\title{
UniStArt: A 12-month prospective observational study of body weight, dietary intake, and physical activity levels in Australian first-year university students
}

\author{
Nina A. Wilson ${ }^{1}$, Anthony Villani ${ }^{1,2}$, Sze-Yen Tan ${ }^{1,3}$ and Evangeline Mantzioris \\ $1, *$ \\ ${ }^{1}$ University of South Australia, Clinical and Health Sciences; \\ nina.wilson@.unisa.edu.au \\ 2 University of the Sunshine Coast, School of Health and Sport Sciences; \\ avillani@usc.edu.au \\ ${ }^{3}$ Deakin University, Institute for Physical Activity and Nutrition (IPAN), School of \\ Exercise and Nutrition Sciences, Geelong, Australia; szeyen.tan@deakin.edu.au \\ * Correspondence: evangeline.mantzioris@ unisa.edu.au; Tel.: +61 883022574
}

Background: Students in the United States gain weight significantly during their first year of university, but limited data is available for Australian students.

Methods: This 12-month observational study was conducted to monitor monthly body weight and body composition, as well as quarterly eating behaviours, dietary intake, physical activity, sedentary behaviours, and basal metabolic rate changes amongst firstyear Australian university students. Participants were first-year university students over 18 years.

Results: Twenty-two first-year university students (5 males, 17 females) completed the study. Female students gained weight significantly at two, three and four-months $(+0.9$ $\mathrm{kg} ;+1.5 \mathrm{~kg} ;+1.1 \mathrm{~kg}, \mathrm{p}<0.05)$. Female waist circumference $(2.5 \mathrm{~cm}$ increase at twomonths, $\mathrm{p}=0.012)$ and body fat also increased $(+0.7 \%, \mathrm{p}=0.04$ at two-months; $+0.9 \%$, $\mathrm{p}=0.026$ at three-months). Intake of sugar, saturated fat (both $>10 \%$ of total energy) and sodium exceeded recommended levels $(>2300 \mathrm{mg}$ ) at 12-months. Greater sedentary behaviours were observed amongst male students throughout the study $(\mathrm{p}<0.05)$. 
Conclusions: Female students are at risk of unfavourable changes in body composition during the first year of university, while males are at risk of increased sedentary behaviours. High intakes of saturated fat, sugars and sodium warrant future interventions in such a vulnerable group.

Keywords: freshman; weight gain; body composition; diet; physical activity 


\section{Introduction}

Overweight and obesity is a prevalent health problem in Australia across all age groups, with $67 \%$ of Australians over the age of 18 overweight or obese [1]. Obesity-related comorbidities include cardiovascular diseases, various cancers, endocrine issues including type two diabetes, and musculoskeletal disorders [2]. The economic impact of the health consequences and co-morbidities of overweight and obesity was reported to be $\$ 58$ billion in 2008 [3], and this is expected to rise significantly in the coming decades.

Research into overweight and obese in young adult populations is scant despite $38.4 \%$ of young Australians aged 18-24 years being overweight or obese [4]. This high prevalence may be due to early adulthood being a time in which there is a considerable change in habitual dietary behaviours and a reduction in physical activity [5-12]. Preventing early onset of excessive weight gain is pertinent for the prevention of excessive adiposity, and associated comorbidities later in life [13]; therefore a better understanding of the aetiology of obesity during this time is required to implement early intervention strategies in this vulnerable group.

Changes in living arrangements and decision making related to lifestyle often starts around early adulthood, a time for which many people also coincides with the commencement of university [14,15]. In Australia, $61 \%$ of newly enrolled domestic university students fall within the age range of 15-24 years [16], an age which overlaps with transitioning to independence in many aspects of life, including lifestyle decisions that may impact on health status. As such, understanding the lifestyle and weight 
changes of the Australian young adult population engaged in tertiary education is important.

In the United States (US), there is a common belief referred to as the 'Freshman 15', where college freshmen (first-year university students, in Australia) would gain $6.8 \mathrm{~kg}$ (15 lbs) during their first year of college [17]. There have been a number of studies designed to investigate this phenomenon in the US. The body of evidence does demonstrate weight gain amongst first-year college students, however this reported weight gain ranges between 0.7 to $4.2 \mathrm{~kg}$, rather than the alleged $6.8 \mathrm{~kg}$ [18-22]. Although this gain is significantly less than the 'Freshman 15' claim, first-year university students are susceptible to weight gain, however, this is yet to be investigated in an Australian population. Findings from the US and other countries are difficult to extrapolate to Australian settings due to differences in living and environmental arrangements. Furthermore, previous studies from the US have not assessed all factors related to body weight regulation via energy intake and energy expenditure simultaneously, and it is unclear how habitual dietary intake, physical activity and other external factors interact and influence the body weight of a university cohort.

This 12-month observational study aimed to monitor changes in body weight among first-year students enrolled in an Australian university and to investigate whether dietary intake and physical activity behaviours, in addition to eating behaviour and basal metabolic rate (BMR), impact on weight change in these students. 


\section{Methods}

The 12-month prospective observational study followed first-year university students during the academic year from March 2015 to February 2016. Ethics approval for the study was obtained in October 2014 from the Human Research Ethics Committee at the University of South Australia (approval number 0000033624) and the study was registered with the Australia New Zealand Clinical Trials Registry (ACTRN12615001116516).

Participants were recruited over a three-week period: during orientation week (final week of February 2015) through to the second week of semester (March 2015). Participants were first-year university students, older than 18 years of age, and had not studied at tertiary level in the previous five years. Additionally, they had no significant medical conditions that could affect key outcome variables of interest i.e. gastrointestinal disorders, eating disorders, renal disease, and cancer.

Participants were recruited via study flyers placed around two university campuses. Flyers were also provided to university student services for all new students collecting orientation packs. Students were also approached during orientation week, and study information emails were also distributed via teaching staff.

Participants who met all inclusion criteria were enrolled into the study. During their first visit, participants were provided with an information sheet and written informed consent was obtained from all participants prior to study commencement.

The study consisted of one baseline visit and twelve monthly follow-up visits. The baseline visit involved all measurements including anthropometric measurements, a three-day diet diary (to be completed before the following visit), a series of 
demographic, physical activity, and eating behaviour questionnaires, and an assessment of basal metabolic rate (BMR). The 12-month visit was identical to the baseline visit.

At three, six, and nine months, all anthropometric measurements were assessed, demographic, physical activity and eating behaviour questionnaires were completed, and participants completed a three-day food diary. During routine, monthly follow-up visits, body weight and body fat percentage were assessed.

Participants' demographics, university enrolment (program, course contact hours, study type), living arrangements including cooking abilities (scored on a 1-100 scale, with 1 being not confident and 100 being very confident) and money spent on groceries, eating out, and foods bought on-campus was obtained through a questionnaire.

Height, weight, waist and hip circumference measurements were conducted by trained personnel according to International Standards for Anthropometry and Kinesiology (ISAK) guidelines [23]. Body weight was recorded to the nearest $0.1 \mathrm{~kg}$ using calibrated digital scales (Tanita BF-679W Scale and Body Fat Monitor, Tanita Inc, Tokyo, Japan) with participants wearing light clothing and without footwear. Height was measured using a portable stadiometer to the nearest $0.1 \mathrm{~cm}$ (Portable Leicester Height Measure, Mk II Stadiometer), with the participant's head positioned in the Frankfort plane. Height and weight were used to calculate Body Mass Index $(\mathrm{BMI}=$ mass $(\mathrm{kg}) / \mathrm{height}$ $\left.\left(\mathrm{m}^{2}\right)\right)$. The Tanita scale is a single-frequency leg-to-leg bioelectrical impedance analysis (SF-BIA) device (Tanita BF 679W Scale and Body Fat Monitor, Tanita Inc, Tokyo, Japan). In accordance with the manufacturer's manual, the subjects stood on the metal contacts in bare feet, and body fat $\%(\mathrm{BF} \%)$ was determined. The measurement was taken in duplicate with the mean value used in the final analysis. Previous literature has 
supported the application of SF-BIA as a portable method of assessing BF\% with its acceptable relative agreement against Dual Energy X-Ray Absorptiometry (DEXA) [24,25]. Waist measurements were taken at the point of the visual narrowing, and hip measurements were taken at the furthest protruding point of the buttocks [23], using a flexible steel measuring tape (Lufkin Executive Thinline flexible steel tape, W606PM). All measures were taken in duplicate, and the mean was calculated. If the measurements exceeded $0.5 \mathrm{~cm}$, a third measurement was taken and the mean of the three measurements was calculated.

Habitual dietary intake was assessed every three months using a three-day food diary. Participants recorded all food and beverage consumption over three days: two days oncampus and one day off-campus. Detailed instructions on how to complete the diet diary and an example of a recorded diary were provided to participants. The researcher verbally cross-checked individual food diaries for completeness and acquired additional information about the reported foods and beverages, as required. Habitual dietary intake was analysed using the dietary analysis software, FoodWorks 8 Professional, using the AusFoods and AusBrands databases [26].

Based on the three-day diet diary, a food group analysis was also performed using the Australian Guide to Healthy Eating (AGHE) food groups as a criterion reference [27]. Using the dietary information provided in the diet diaries, each food and beverage item was categorised into food groups based on the type of food and the nutrient profile. The weight of the food and/or beverage was converted into a common serving size of that food, as outlined by the AGHE [27]. All data was entered into an Excel spreadsheet and the mean serve size for each food group was calculated for on-campus university days, 
off-campus days, and for the total three days. Average serve size consumption was also calculated for the total cohort at each time-point.

The validated 27-item International Physical Activity Questionnaire (IPAQ) [28] was used to estimate average weekly physical activity. This questionnaire acquired information on the types of vigorous, moderate, and sedentary physical activities undertaken in the last seven days. The questionnaire was divided into five categories: 1) job-related physical activity, 2) transportation physical activity, 3) housework, house maintenance and caring for family, 4) recreation, sport, and leisure-time physical activity, and 5) time spent sitting. The IPAQ was scored using the associated scoring document provided, and reported as total Metabolic Equivalent of Task (MET) minutes/week. This is a measure that identifies the energy expenditure of certain physical activities, and was used as the scoring value for this questionnaire [29].

BMR was measured using a previously validated indirect calorimeter method [30] that assessed oxygen and carbon dioxide gas exchanges at rest (TrueOne 2400 Metabolic Measurement System, Parvomedics Inc., USA). The coefficient of variation of indirect calorimeter measurements used in this study was $10.5 \%$. The measurement was taken after a 10-12 hour fast, with no alcohol and strenuous exercise on the day prior to assessment. Upon arrival, participants rested for 15-minutes in a seated position. After the resting period, a ventilated hood was placed over the upper body of participants, and gas samples were analysed continuously for 30-minutes. As per standard REE measurement protocol, only the last 20-minutes of the data from the 30-minute measurement period were used for analysis. The first 10-minutes were considered a habituation period and this data was discarded. During the measurement period, using the published Weir equation[31], the BMR measurement was calculated based on the 
volume of oxygen consumed $\left(\mathrm{VO}_{2}\right)$ and the volume of carbon dioxide produced $\left(\mathrm{VCO}_{2}\right)$.

The validated Three-Factor Eating Questionnaire (TFEQ)[32] was used to characterise individuals' eating behaviours: cognitive restraint, disinhibition and hunger. The score range for each behaviour was 0-21 for cognitive restraint, 0-16 for disinhibition and 014 for hunger.

Statistical analyses were conducted using IBM Statistical Product and Service Solution software (Chicago, USA)[33]. Due to the high attrition rate of $24 \%$ in our study, an 'intention-to-treat' (ITT) analysis was first performed on all enrolled participants, where missing data was tabulated using a 'previous observation carried forward' method. Following that, a 'per protocol' (PP) analysis was also conducted using completers' data only. All variables were tested for normality to determine parametric or non-parametric analyses. Multiple linear regressions were run to ensure assumptions of multicollinearity and homoscedasticity were not violated. Chi-square goodness of fit tests $\left(\chi^{2}\right)$ were used to compare categorical demographic responses. General Linear Model (GLM) for repeated measures ANOVA with Bonferroni correction was used to compare means between study time points (months) on the total sample, and paired samples t-tests were used to identify changes from baseline. All data are presented as mean \pm standard deviation (SD), and statistical significance was set at $P<0.05$. 


\section{Results}

Eighty-eight participants expressed interest in the study, where 29 participants were eligible and recruited into the study. Of the 29 participants who commenced, 22 participants ( $\mathrm{n}=5$ males; $\mathrm{n}=17$ females) completed the study at the 12 -month time point (Figure 1).

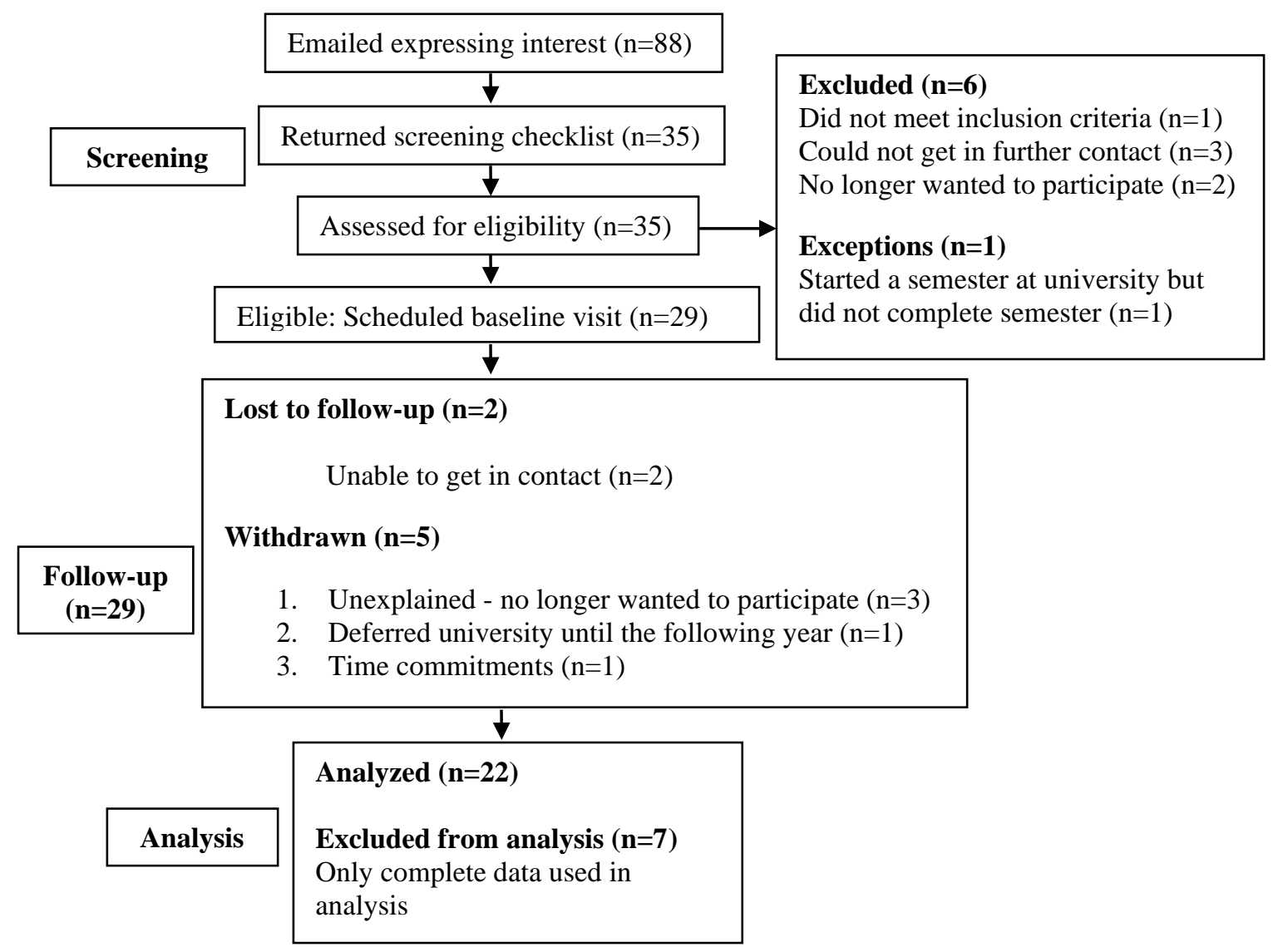

Figure 1. Consort chart outlining the UniStArt study process from participant screening and recruitment until analysis. A total of 29 participants enrolled in the study, while 22 participants were used for analysis.

Baseline characteristics of all participants as well as those who completed the study are presented in Table 1. The proportion of male-to-female students and the baseline characteristics of all $(n=29)$ and completers $(n=22)$ were not significantly different. 
Table 1. Baseline demographics and characteristics of 22 first-year university students from an Australian university.

\begin{tabular}{|c|c|}
\hline Characteristics $(n=22)$ & Mean \pm SD \\
\hline \multicolumn{2}{|l|}{ Clinical characteristics } \\
\hline $\operatorname{Sex}(M / F)$ & 5 males \& 17 females \\
\hline Age (years) & $21.1 \pm 6.8$ \\
\hline Height $(\mathrm{cm})$ & $167.6 \pm 9.3$ \\
\hline Weight (kg) & $65.0 \pm 19.2$ \\
\hline Body Fat (\%) & $24.0 \pm 9.2$ \\
\hline Body Mass Index $\left(\mathrm{kg} / \mathrm{m}^{2}\right)$ & $22.9 \pm 5.4$ \\
\hline Waist Circumference (cm) & $75.2 \pm 13.2$ \\
\hline Hip Circumference (cm) & $97.9 \pm 11.9$ \\
\hline Basal Metabolic Rate (Kcal/day) & $1392 \pm 376$ \\
\hline Demographic characteristics & n (\%) \\
\hline \multicolumn{2}{|l|}{ Marital status } \\
\hline Single & $20(90.9 \%)$ \\
\hline Married & $1(4.5 \%)$ \\
\hline Divorced & $0(0 \%)$ \\
\hline De-facto & $1(4.5 \%)$ \\
\hline \multicolumn{2}{|l|}{ Smoking status } \\
\hline Current smoker & $1(4.5 \%)$ \\
\hline Non-smoker & $20(90.9 \%)$ \\
\hline Smoked previously & $1(4.5 \%)$ \\
\hline \multicolumn{2}{|l|}{ Employment status } \\
\hline Employed & $8(36.4 \%)$ \\
\hline \multicolumn{2}{|l|}{ Living situation prior to university } \\
\hline Alone & $1(4.5 \%)$ \\
\hline With parents & $18(81.8 \%)$ \\
\hline With partner & $2(9.1 \%)$ \\
\hline With friends (rent a room) & $1(4.5 \%)$ \\
\hline Residential college & $0(0 \%)$ \\
\hline \multicolumn{2}{|l|}{ Current living situation } \\
\hline Alone & $2(9.1 \%)$ \\
\hline With parents & $13(59.1 \%)$ \\
\hline With partner & $4(18.2 \%)$ \\
\hline With friends (rent a room) & $2(9.1 \%)$ \\
\hline Residential college & $1(4.5 \%)$ \\
\hline \multicolumn{2}{|c|}{ Pre-university vs. current living situation ${ }^{(a)}, p=0.515$} \\
\hline \multicolumn{2}{|l|}{ Enrolment type } \\
\hline Full-time $(n=20)$ & 90.9 \\
\hline Part-time $(n=2)$ & 9.1 \\
\hline \multicolumn{2}{|l|}{ Grocery shopping } \\
\hline Myself $(n=5)$ & 22.7 \\
\hline Parents/partner/housemate $(n=16)$ & 72.7 \\
\hline Homestay/residential college $(n=1)$ & 4.5 \\
\hline
\end{tabular}

(a) Chi-square test. $\mathrm{P}$ value suggests that living arrangement prior to vs. after university commencement remained unchanged. 
Throughout the first year at university, monthly body weight changed over time in the total sample but was not statistically significant (Figure 2). However, weight change observed in males compared to females followed different trajectories, specifically, significant weight gain from baseline was observed in females from two to four months $(\mathrm{p}=0.004, \mathrm{p}=0.006, \mathrm{p}=0.037)$. Towards the end of the study, body weight normalized to baseline weight.

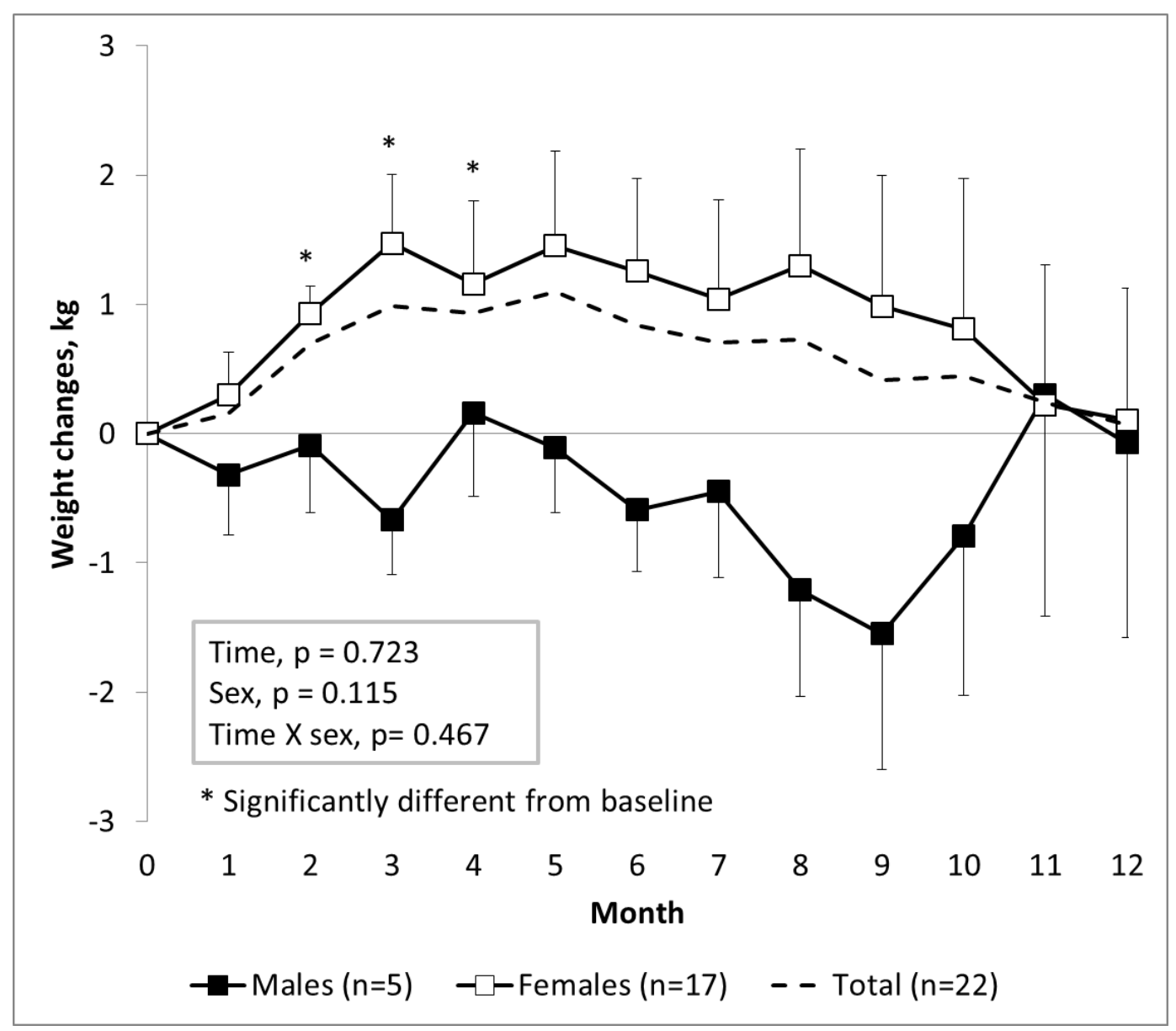

* Significantly different from baseline, paired-sample t-tests, $\mathrm{p}<0.05$

Figure 2. Different trajectories of monthly body weight change between male $(n=5)$ and female $(n=17)$ students during their first year at university. Female students are at risk for weight gain during the first few months as compared to their male counterparts. 
The ITT and PP analysis of all study outcomes are presented in Table 2 . The use of either statistical analysis methods did not change the findings of our study. Furthermore, since this was an observational study, non-compliance to treatment is not a main concern, and therefore the as per protocol analysis of 22 study completers was performed and reported in this manuscript.

Table 2. Anthropometric, physical activity, dietary intake, and eating behaviour in 22 students during their first year at an Australian university.

\begin{tabular}{|c|c|c|c|c|c|c|c|c|}
\hline & $\begin{array}{c}\text { Baseline } \\
\text { Mean } \pm \text { SD }\end{array}$ & $\begin{array}{c}\text { 3-months } \\
\text { Mean } \pm \text { SD }\end{array}$ & $\begin{array}{c}\text { 6-months } \\
\text { Mean } \pm \text { SD }\end{array}$ & $\begin{array}{c}\text { 9-months } \\
\text { Mean } \pm \text { SD }\end{array}$ & $\begin{array}{l}\text { 12-months } \\
\text { Mean } \pm \text { SD }\end{array}$ & $\begin{array}{c}\text { Time, } \\
\text { p }\end{array}$ & $\begin{array}{c}\text { Sex, } \\
\text { p }\end{array}$ & $\begin{array}{c}\text { Time X } \\
\text { sex, p }\end{array}$ \\
\hline \multicolumn{9}{|l|}{ Anthropometry } \\
\hline Weight, kg & $65.0 \pm 19.2$ & $66.0 \pm 19.8^{(\mathbf{a})}$ & $65.8 \pm 19.7$ & $65.4 \pm 19.9$ & $65.1 \pm 20.1$ & \multirow{3}{*}{0.723} & \multirow{3}{*}{0.115} & \multirow{3}{*}{0.467} \\
\hline$M(n=5)$ & $78.3 \pm 30.1$ & $77.6 \pm 30.8$ & $77.7 \pm 29.5$ & $76.7 \pm 28.0$ & $78.2 \pm 28.4$ & & & \\
\hline $\mathrm{F}(\mathrm{n}=17)$ & $61.1 \pm 13.6$ & $62.6 \pm 15.0^{(\mathbf{a})}$ & $62.4 \pm 15.3$ & $62.1 \pm 16.5$ & $61.2 \pm 16.1$ & & & \\
\hline Body fat, $\%$ & $24.0 \pm 9.2$ & $25.3 \pm 10.4$ & $24.3 \pm 9.6$ & $24.0 \pm 9.2$ & $24.2 \pm 9.2$ & \multirow{3}{*}{0.199} & \multirow{3}{*}{$0.033^{*}$} & \multirow{3}{*}{0.381} \\
\hline$M(n=5)$ & $16.9 \pm 9.1$ & $19.3 \pm 15.5$ & $16.4 \pm 9.1$ & $16.2 \pm 9.1^{(\mathbf{a})}$ & $17.5 \pm 8.7$ & & & \\
\hline $\mathrm{F}(\mathrm{n}=17)$ & $26.1 \pm 8.4$ & $27.0 \pm 8.3^{(\mathbf{a})}$ & $26.6 \pm 8.7$ & $26.2 \pm 8.1$ & $26.2 \pm 8.6$ & & & \\
\hline Waist, cm & $75.2 \pm 13.2$ & $76.8 \pm 12.2$ & $76.4 \pm 12.8$ & $75.9 \pm 12.9$ & $76.0 \pm 13.2$ & \multirow{3}{*}{0.855} & \multirow{3}{*}{0.167} & \multirow{3}{*}{0.235} \\
\hline$M(n=5)$ & $83.9 \pm 17.8$ & $82.2 \pm 19.0$ & $82.7 \pm 18.4$ & $82.3 \pm 17.5$ & $83.9 \pm 17.1$ & & & \\
\hline $\mathrm{F}(\mathrm{n}=17)$ & $72.7 \pm 10.9$ & $75.2 \pm 9.6^{(\mathbf{a})}$ & $74.6 \pm 10.7$ & $74.0 \pm 11.1$ & $73.7 \pm 11.4$ & & & \\
\hline Hip, cm & $97.9 \pm 11.9$ & $99.1 \pm 12.2$ & $99.3 \pm 12.8$ & $98.2 \pm 13.3$ & $97.3 \pm 13.3$ & \multirow{3}{*}{0.591} & \multirow{3}{*}{0.698} & \multirow{3}{*}{0.310} \\
\hline$M(n=5)$ & $100.7 \pm 12.8$ & $12.2 \pm 12.6$ & $100.1 \pm 13.2$ & $100.0 \pm 11.7$ & $100.5 \pm 11.4$ & & & \\
\hline$F(n=17)$ & $97.1 \pm 11.9$ & $98.7 \pm 12.5$ & $99.1 \pm 13.1$ & $97.6 \pm 14.0$ & $96.3 \pm 14.0$ & & & \\
\hline \multicolumn{9}{|l|}{ Dietary intake } \\
\hline Energy intake, kJ & $8915 \pm 2437$ & $9245 \pm 2978$ & $8532 \pm 2862$ & $7679 \pm 2393^{(a)}$ & $7606 \pm 2060^{(\mathbf{a})}$ & \multirow{3}{*}{0.073} & \multirow{3}{*}{$0.001 * * *$} & \multirow{3}{*}{0.830} \\
\hline$M(n=5)$ & $10339 \pm 3687$ & $11969 \pm 2575$ & $10825 \pm 1822$ & $9305 \pm 3513$ & $9584 \pm 2465$ & & & \\
\hline$F(n=17)$ & $8496 \pm 1892$ & $8444 \pm 2643$ & $7857 \pm 2791$ & $7201 \pm 1834^{(\mathbf{a})}$ & $7024 \pm 1572^{(\mathbf{a})}$ & & & \\
\hline Protein intake, $g$ & $85.3 \pm 31.5$ & $94.9 \pm 32.7$ & $90.3 \pm 32.5$ & $79.0 \pm 29.9$ & $83.0 \pm 30.7$ & \multirow{3}{*}{0.095} & \multirow{3}{*}{0.126} & \multirow{3}{*}{0.568} \\
\hline$M(n=5)$ & $91.5 \pm 34.7$ & $115.9 \pm 31.1^{(\mathbf{a})}$ & $113.9 \pm 18.2$ & $85.2 \pm 35.0$ & $96.3 \pm 31.9$ & & & \\
\hline $\mathrm{F}(\mathrm{n}=17)$ & $83.5 \pm 31.3$ & $88.7 \pm 31.3$ & $83.5 \pm 32.9$ & $77.1 \pm 29.2$ & $79.1 \pm 30.1$ & & & \\
\hline Fat intake, $\mathrm{g}$ & $85.3 \pm 29.2$ & $82.3 \pm 27.7$ & $74.4 \pm 25.8$ & $65.9 \pm 23.0^{(\mathbf{a})}$ & $64.5 \pm 20.1^{(\mathbf{a})}$ & \multirow{3}{*}{$0.031^{*}$} & \multirow{3}{*}{0.184} & \multirow{3}{*}{0.990} \\
\hline$M(n=5)$ & $96.6 \pm 46.1$ & $89.1 \pm 33.2$ & $84.0 \pm 13.1$ & $71.0 \pm 32.7$ & $71.1 \pm 16.2$ & & & \\
\hline $\mathrm{F}(\mathrm{n}=17)$ & $81.9 \pm 23.1$ & $80.3 \pm 26.8$ & $71.6 \pm 28.2$ & $64.4 \pm 20.5^{(\mathbf{a})}$ & $62.5 \pm 21.1^{(\mathrm{a})}$ & & & \\
\hline Saturated fat intake, $g$ & $30.7 \pm 10.4$ & $30.7 \pm 11.9$ & $26.9 \pm 12.6$ & $23.3 \pm 8.5^{(\mathrm{a})}$ & $23.1 \pm 9.4^{(\mathbf{a})}$ & \multirow{3}{*}{0.083} & & \\
\hline$M(n=5)$ & $34.3 \pm 13.1$ & $33.9 \pm 13.4$ & $31.4 \pm 11.2$ & $25.1 \pm 10.5$ & $28.0 \pm 5.3$ & & 0.128 & 0.983 \\
\hline $\mathrm{F}(\mathrm{n}=17)$ & $29.7 \pm 9.6$ & $29.7 \pm 11.6$ & $25.6 \pm 12.9$ & $22.8 \pm 8.2^{(\mathbf{a})}$ & $21.7 \pm 10.0^{(\mathbf{a})}$ & & & \\
\hline CHO intake, $g$ & $242.2 \pm 77.4$ & $260.6 \pm 111.4$ & $240.0 \pm 91.6$ & $218.5 \pm 81.8$ & $211.5 \pm 80.9$ & & & \\
\hline$M(n=5)$ & $290.8 \pm 103.0$ & $385.6 \pm 108.1$ & $329.4 \pm 80.7$ & $292.5 \pm 109.3$ & $299.1 \pm 120.8$ & 0.122 & $<0.001 * * *$ & 0.445 \\
\hline$F(n=17)$ & $227.9 \pm 65.3$ & $223.8 \pm 84.0$ & $213.8 \pm 78.4$ & $196.7 \pm 59.8$ & $185.8 \pm 42.9^{(\mathbf{a})}$ & & & \\
\hline Sugar intake, g & $96.1 \pm 34.0$ & $90.1 \pm 35.9$ & $87.9 \pm 30.6$ & $84.4 \pm 35.2$ & $71.0 \pm 27.7^{(\mathbf{a})}$ & & & \\
\hline$M(n=5)$ & $113.8 \pm 51.1$ & $122.1 \pm 18.9$ & $92.5 \pm 31.4$ & $98.6 \pm 43.4$ & $78.0 \pm 32.2$ & $0.028^{*}$ & 0.081 & 0.397 \\
\hline $\mathrm{F}(\mathrm{n}=17)$ & $90.9 \pm 27.1$ & $80.7 \pm 34.5$ & $86.6 \pm 31.2$ & $80.2 \pm 32.7$ & $69.0 \pm 26.9^{(\mathbf{a})}$ & & & \\
\hline Sodium, mg & $2440 \pm 767$ & $3029 \pm 1558$ & $2827 \pm 1449$ & $2673 \pm 2140$ & $2542 \pm 953$ & & & \\
\hline$M(h=5)$ & $2775 \pm 959$ & $4717 \pm 1344^{(\mathbf{a})}$ & $3673 \pm 1134$ & $3063 \pm 1994$ & $3219 \pm 406$ & 0.115 & $-0.042 *$ & 0.241 \\
\hline$F(n=17)$ & $2341 \pm 704$ & $2532 \pm 1258$ & $2579 \pm 1465$ & $2558 \pm 2226$ & $2342 \pm 983$ & & & \\
\hline Physical activity & & & & & & & & \\
\hline PA, MET mins & $3704 \pm 3260$ & $2896 \pm 2301$ & $4824 \pm 3479$ & $5897 \pm 9910$ & $3770 \pm 1840$ & & & \\
\hline$M(n=5)$ & $5985 \pm 2197$ & $3688 \pm 1782$ & $7481 \pm 3531$ & $7282 \pm 8787$ & $3989 \pm 1270$ & 0.265 & 0.258 & 0.645 \\
\hline $\mathrm{F}(\mathrm{n}=17)$ & $3033 \pm 3263$ & $2662 \pm 2430$ & $4042 \pm 3149$ & $5490 \pm 10432$ & $3705 \pm 2005$ & & & \\
\hline Sitting time, $\mathrm{min} / \mathrm{wk}$ & $2360 \pm 912$ & $2527 \pm 1095$ & $2729 \pm 1007$ & $2340 \pm 1066$ & $2663 \pm 1070$ & 0.090 & 0.827 & $0.037^{*}$ \\
\hline
\end{tabular}




\begin{tabular}{|c|c|c|c|c|c|c|c|c|}
\hline$M(n=5)$ & $1944 \pm 757$ & $2172 \pm 1170$ & $2712 \pm 1187$ & $2676 \pm 1383$ & $3444 \pm 1209^{(a)}$ & & & \\
\hline$F(n=17)$ & $2483 \pm 937$ & $2631 \pm 1086$ & $2734 \pm 989$ & $2241 \pm 983$ & $2434 \pm 944$ & & & \\
\hline \multicolumn{9}{|c|}{ Basal metabolic rate } \\
\hline $\mathrm{BMR}, \mathrm{kcal} / \mathrm{d}$ & $1392 \pm 376$ & & & & $1293 \pm 338$ & & & \\
\hline$M(n=5)$ & $1817 \pm 458$ & Not measured & Not measured & Not measured & $1613 \pm 503$ & $0.001 * * *$ & $0.003 * *$ & 0.062 \\
\hline$F(n=16)$ & $1259 \pm 230$ & & & & $1193 \pm 201$ & & & \\
\hline \multicolumn{9}{|c|}{ Eating behaviours } \\
\hline Restraint & $8.2 \pm 5.1$ & $8.0 \pm 5.7$ & $7.2 \pm 5.4$ & $7.7 \pm 5.3$ & $7.4 \pm 5.0$ & & & \\
\hline$M(n=5)$ & $6.2 \pm 3.6$ & $5.0 \pm 2.1$ & $4.6 \pm 2.7$ & $4.2 \pm 1.6$ & $4.4 \pm 1.5$ & 0.347 & 0.159 & 0.722 \\
\hline$F(n=16)$ & $8.8 \pm 5.4$ & $8.9 \pm 6.1$ & $7.9 \pm 5.9$ & $8.7 \pm 5.6$ & $8.3 \pm 5.4$ & & & \\
\hline Hunger & $5.5 \pm 3.1$ & $5.8 \pm 3.5$ & $6.0 \pm 3.6$ & $5.8 \pm 3.1$ & $5.5 \pm 3.6$ & & & \\
\hline$M(n=5)$ & $8.0 \pm 2.9$ & $8.8 \pm 3.6$ & $9.4 \pm 3.8$ & $8.2 \pm 3.1$ & $9.0 \pm 4.3$ & 0.671 & $0.013^{*}$ & 0.548 \\
\hline$F(n=16)$ & $4.8 \pm 2.8$ & $4.9 \pm 3.0$ & $5.0 \pm 3.0$ & $5.1 \pm 2.9$ & $4.5 \pm 2.8$ & & & \\
\hline Disinhibition & $5.6 \pm 2.4$ & $6.0 \pm 2.6$ & $6.0 \pm 3.1$ & $5.4 \pm 2.6$ & $5.3 \pm 2.8$ & & & \\
\hline$M(n=5)$ & $5.6 \pm 2.4$ & $5.2 \pm 3.3$ & $7.0 \pm 4.4$ & $4.6 \pm 3.4$ & $5.2 \pm 3.8$ & 0.264 & 0.879 & 0.190 \\
\hline$F(n=16)$ & $5.6 \pm 2.5$ & $6.3 \pm 2.5$ & $5.6 \pm 2.6$ & $5.7 \pm 3.4$ & $5.3 \pm 2.6$ & & & \\
\hline
\end{tabular}

PA - physical activity; CHO - carbohydrate; BMR - basal metabolic rate

(a) Significantly from baseline values, paired-sample t-tests, $\mathrm{p}<0.05$

* Significant effects, general linear model for repeated measures ANOVA, $\mathrm{p}<0.05$

** Significant effects, general linear model for repeated measures ANOVA, $p<0.01$

*** Significant effects, general linear model for repeated measures ANOVA, $\mathrm{p}<0.001$

There was no significant change in body fat $\%$ in the total sample, however there were significant sex effects $(\mathrm{p}=0.033)$. Consistent with weight change patterns in females, body fat was significantly higher at baseline, two, three and five-months and waist circumference was significantly higher at three-months than at baseline. BMR was higher in males than in females (sex effects, $p=0.003$ ) and reduced over 12-months (time effects, $\mathrm{p}=0.001$ ); this decrease in BMR between sexes approached statistical significance (interaction effects, $\mathrm{p}=0.062$ ).

Mean daily energy intake significantly decreased from baseline in the total sample at 9and 12-months $(\mathrm{p}<0.05)$, and was predominantly attributed to a significant reduction in energy intake in females at these time points (sex effects, $\mathrm{p}=0.001$ ). Lower total energy intake was also contributed to by a significant reduction in total fat, saturated fat, carbohydrate, and sugar consumption. Conversely male students did not alter dietary intake significantly, but sodium intake significantly increased at 12-months (sex effects, 
$\mathrm{p}=0.042$ ). Total daily sugar, saturated fat and sodium intakes exceeded recommended levels (less than $10 \%$ of total energy intake for saturated fat and sugar, and an upper limit of 2300mg of sodium) in all participants.

No significant changes in eating behaviour scores was observed, but hunger was found to be higher in males than in females ( relatively stable in all participants, but a significant increase in sitting time was observed in male students at 12-months of the study (interaction effects, $\mathrm{p}=0.037$ ).

The food group analysis (Table 3) indicated that at 12-months there was a significant reduction in discretionary sweet food intake $(\mathrm{p}=0.03)$. Analyses further revealed that students did not eat differently between university and non-university days. Moreover, dietary intake remained unchanged throughout the study, except for a significant reduction in discretionary sweet food intake at 12 -months $(\mathrm{p}=0.03)$.

When compared with the AGHE recommendations, participants consumed less than the recommended daily serves for wholegrain cereals, fruit, vegetables, protein foods, and milk and milk alternatives throughout the 12-months. Consumption of savoury discretionary foods exceeded recommendations at baseline and 6-months $(4.5 \pm 3.5$ and $3.9 \pm 4.2$ serves/day respectively). 
Table 3. Food group analyses from the diet diaries of $n=22$ university students during their first year at an Australian university.

\begin{tabular}{|c|c|c|c|c|c|c|c|c|c|}
\hline \multirow{2}{*}{ Food Group } & \multirow{2}{*}{ AGHE Serving Size } & \multicolumn{2}{|c|}{ Baseline } & \multicolumn{2}{|c|}{ 6-months } & \multicolumn{2}{|c|}{ 12-months } & \multirow{2}{*}{$\begin{array}{c}\text { Time } \\
\mathbf{p}\end{array}$} & \multirow{2}{*}{$\begin{array}{c}\text { Interaction } \\
\mathbf{p}\end{array}$} \\
\hline & & Mean & SD & Mean & SD & Mean & SD & & \\
\hline Bread \& cereals & $30-125 \mathrm{~g}$ & 3.9 & 2.4 & 3.5 & 1.8 & 3.5 & 1.8 & 0.844 & 0.268 \\
\hline Fruit & $150 \mathrm{~g}$ & 0.9 & 1.1 & 0.8 & 0.7 & 0.5 & 0.6 & 0.205 & 0.428 \\
\hline Processed fruit & $30 \mathrm{~g} / 125 \mathrm{ml}$ & 0.5 & 0.8 & 0.4 & 0.6 & 0.2 & 0.5 & 0.351 & 0.173 \\
\hline Fresh/frozen veg & $75 \mathrm{~g}$ & 2.8 & 2.3 & 3 & 2.2 & 2.6 & 2 & 0.864 & 0.571 \\
\hline Canned veg & $75 \mathrm{~g}$ & 0.2 & 0.4 & 0.2 & 0.4 & 0.3 & 0.5 & 0.847 & 0.468 \\
\hline Legumes & $150 \mathrm{~g}$ & 0.1 & 0.2 & 0.1 & 0.2 & 0 & 0.1 & 0.407 & 0.07 \\
\hline Milk \& alternatives & $40-250 \mathrm{~g} / \mathrm{ml}$ & 1.1 & 0.9 & 1 & 0.7 & 1 & 0.8 & 0.894 & 0.734 \\
\hline Low-fat milk & $40-250 \mathrm{~g} / \mathrm{ml}$ & 0.3 & 0.6 & 0.2 & 0.3 & 0.2 & 0.3 & 0.129 & 0.743 \\
\hline Meat \& alternatives & $65-80 \mathrm{~g}$ & 0.4 & 0.7 & 0.6 & 0.7 & 0.6 & 0.4 & 0.49 & 0.609 \\
\hline Fatty meat & 60g (processed)-65g & 0.2 & 0.3 & 0.3 & 0.6 & 0.1 & 0.2 & 0.389 & 0.464 \\
\hline Lean meat/Poultry & $65 \mathrm{~g}$ lean $/ 80 \mathrm{~g}$ poultry & 1 & 1.2 & 0.9 & 0.8 & 1.1 & 1.6 & 0.978 & 0.152 \\
\hline Fish \& seafood & $100 \mathrm{~g}$ & 0.3 & 0.4 & 0.4 & 0.5 & 0.2 & 0.3 & 0.108 & 0.134 \\
\hline Eggs & 2 large eggs $(120 g)$ & 0.2 & 0.3 & 0.1 & 0.2 & 0.1 & 0.2 & 0.14 & 0.566 \\
\hline Nuts \& seeds & $30 \mathrm{~g}$ & 0.2 & 0.4 & 0.2 & 0.4 & 0.2 & 0.4 & 0.792 & 0.596 \\
\hline Unsaturated oils & $5 g$ & 2.5 & 3.2 & 1.8 & 1.9 & 1.7 & 1.8 & 0.293 & 0.255 \\
\hline Alcohol & $\begin{array}{l}100 \mathrm{ml} \text { wine } \\
285 \mathrm{ml} \text { full strength } \\
\text { beer } \\
60 \mathrm{ml} \text { port or sherry } \\
30 \mathrm{ml} \text { spirits }\end{array}$ & 0.2 & 0.6 & 0.1 & 0.3 & 0.2 & 0.4 & 0.309 & 0.079 \\
\hline Discretionary sweet & $600 \mathrm{~kJ}$ & 2.1 & 1.4 & 1.7 & 1.9 & 1 & 1 & $0.026 *$ & 0.972 \\
\hline Discretional savoury & $600 \mathrm{~kJ}$ & 2.4 & 2.1 & 2.2 & 2.3 & 1.9 & 2.2 & 0.587 & 0.582 \\
\hline Soft drink & $375 \mathrm{ml}$ & 0.2 & 0.5 & 0.3 & 0.5 & 0.4 & 0.6 & 0.345 & 0.947 \\
\hline Water & $2600 \mathrm{ml}$ & 1360 & 627 & 1101 & 811 & 1228 & 903 & 0.069 & 0.96 \\
\hline Tea/Coffee & Not stated & 99 & 210 & 71 & 280 & 109 & 249 & 0.298 & 0.903 \\
\hline
\end{tabular}

* Significant effects, general linear model for repeated measures ANOVA, $\mathrm{p}<0.05$ 


\section{Discussion}

To the best of our knowledge, this is the first Australian study investigating the dynamic interactions between body weight, dietary intake, physical activity levels, and eating behaviours of first-year undergraduate university students for 12-months. We observed that female Australian first-year university students are at risk of unfavourable body composition changes, specifically favouring an increase in central adiposity. Male students' body weight reduced, and returned to baseline values again at the end of the study, possibly due to increased sedentary behaviours. Whether these changes in body composition apply to first-year students only or to all students should be investigated in a larger sample to identify whether different strategies may be required to help male and female students maintain a healthy body weight.

Differential weight change trajectories between male and female students was an interesting finding. Weight gain in females was consistent with findings from previous studies in the US, United Kingdom, and Belgium, in which weight and fat mass both increased in first-year university students over the first few months of the academic year [34-36]. However, why this was not observed in male students in our sample warrants further investigations, although the small sample size of males in this study may have influenced our ability to identify significant changes. It is also important to note that all students' weight returned to baseline weight by 12-months, which highlights the importance of regular surveillance of body weight in such studies. Several other studies that have identified significant weight gain in this population have only conducted observations over short periods of time (e.g. three-months or less) and therefore may not have captured periods later on in the year in which students' body weight regulated and returned to baseline [35,37-39]. Findings from previous research have also indicated an 
overall decrease in energy intake, and total fat intake in first-year university students $[18,40-42]$ but this was observed only in female students in our study. The time in which body weight normalised in females was around the time of university breaks in the Summer; this may indicate that females have more time during breaks to maintain a healthy lifestyle, which is in contrast to previous literature that suggests that females gain weight over university holiday-break periods [43]. Additionally, stress during semester and exam times may have resulted in weight gain amongst females [44-47].

There was no significant change in male students' body weight, although weight appeared to decrease and was later regained, resulting in males returning to baseline weight. This regain in body weight may have been attributed to an increase in sitting time at the 12-month study time-point and the associated decrease in BMR observed amongst males. Significant increases in sedentary behaviours have only been observed in two previous studies, and these increases occurred in males also [47,48]. Greater sitting time in males in the present study may be due to the general university lifestyle, as participants became more accustomed to spending time sitting in lectures, tutorials, and studying, but why this increase was shown in males only is unknown. This finding is concerning as sedentary behaviours, independent of physical activity, are associated with increased risks of weight gain, cardiovascular diseases, type two diabetes and overall mortality risk [49-52].

The maintenance of body weight in males and females in this cohort may suggest that the impact of the transition from high school to university in an Australian population is not as strong as previously thought. Differences in the university culture and environments between countries, in which many Australian students attend local universities and hence remain living at home with parents, may be accountable. This 
was the case in our study, as the majority of participants lived at home both prior to, and during, university and this did not significantly change. Importantly, most of the participants specified that their parents, partner, or housemate undertook the grocery shopping, and thus dietary intake was still largely influenced by family and friends. Previous evidence has highlighted potential dietary influences when living at home and suggests that those students who move away from home during university have increasingly poorer dietary quality $[15,53,54]$. Previous evidence has also suggested that those students living on-campus gain more weight than those living off-campus [55] and that peer influence may also be a factor in weight changes during university [56,57].

Although previous studies have reported different eating behaviour patterns among first year university students [58,59], we did not observe this in our study. However, dietary intakes in this study exceeded current guidelines for saturated fat, sugar and sodium [6062]. Previous research in the US has similarly indicated a cause for concern in the diet quality of first-year university students [18] and given the high levels of saturated fat, sugar and sodium consumed in this sample, further analysis of dietary patterns in this vulnerable cohort is warranted. This is particularly important as prolonged exposure to high intake of sodium, sugar and saturated fat may increase the risk of serious health problems, including cardiovascular diseases [63-65]. Early identification of these increased intake levels may warrant early intervention in preventing obesity and other health problems later in life.

The food group analysis in the present study also highlighted an inadequate intake of dairy, fruit and vegetables. These findings are consistent with previous literature in university students from other countries $[9,56,66,67]$ and are of concern as low intakes of 
fruit and vegetables are associated with cardiometabolic disease risk including cardiovascular disease and type 2 diabetes.

This study has a number of strengths. First, it was a prospective observational study allowing for regular surveillance of variables in three main domains: 1) anthropometric measurements, 2) physical activity, and 3) dietary habits, as well as eating behaviours and BMR of first-year university students. For this reason, findings allowed the assessment of how these three aspects interacted to regulate body weight. Despite the small sample size, the long observation period was able to provide insight into weight changes over time and identified that weight returned to normal; an important factor that shorter studies may have overlooked. Results from this study will also provide useful data for future follow-up studies and serve as a basis for consideration for future interventions in Australian universities.

There were also limitations to this research. Firstly, there was a small sample size of 22 participants. The small sample size was due to difficulties in recruitment and the short recruitment period, which was the result of the time constraints involved in capturing the academic year; future studies should consider this for recruitment purposes. Moreover, increases in body fat percentage observed in the study may be the result of violation of the principals of the BIA methodology relating to day-to-day variations in the hydration status and timing of the body composition assessments [68]; nevertheless, a coefficient of variation under repeated measures was performed to minimize this possibility and ensured the reliability of the BIA method in this study (variation of 1.0\%). A small sample size may have limited the statistical power to perform further analyses based on various demographic characteristics; in future, a larger sample size could improve the significance and relevance of the results. Meanwhile, the majority of 
the participants were students at one university campus $(n=21)$, a campus in which many of the programs of study are health-related, highlighting potential issues with the generalisability of the study findings. Thus, it is possible that participants involved in the study may have been more likely to participate in such a study as it was in line with their health and future career interests. In turn, this may have influenced their dietary and physical activity habits and participants may also have monitored their weight more closely than the average university student because of their interests. Future studies should therefore increase the sample size, include students from all faculties, and perhaps also include or continue to follow students from second or third years to determine if weight and lifestyle changes are unique in first-year students only.

\section{Conclusions}

This observational study of first-year Australian university students demonstrated that female students may be at risk for weight gain during the first few months of the academic year, but our findings did not support the "Freshman 15" phenomenon from the US. An increase in sedentary behaviours was also observed in males, and all students exceeded the recommended limits for saturated fat, sugar, and sodium intakes, which warrant further investigation into early nutrition intervention in this vulnerable population.

Acknowledgments: The authors would like to thank BUPA for providing compensatory gifts to participants, Louise Massie for her involvement in organizing participant visits, and the Alliance for Research in Exercise, Nutrition and Activity (ARENA) for providing their facility for the research. 


\section{References}

1. Australian Bureau of Statistics. National Health Survey: first results, 2017-18. Australian Bureau of Statistics: Canberra, 2018.

2. Guh, D.; Zhang, W.; Bansback, N.; Amarsi, Z.; Birmingham, C.L.; Anis, A. The incidence of co-morbidities related to obesity and overweight: A systematic review and meta-analysis. BioMed Central Public Health 2009, 9, 1-20.

3. Australian Bureau of Statistics. Overweight/Obesity; Canberra, 2013.

4. Australian Bureau of Statistics. Overweight and Obesity; Canberra, 2013.

5. Bray, S.R.; Born, H.A. Transition to University and Vigorous Physical Activity: Implications for Health and Psychological Well-Being. Journal of American College Health 2004, 52, 181-188, doi:10.3200/JACH.52.4.181-188.

6. $\quad$ Leslie, E.; Owen, N.; Salmon, J.; Bauman, A.; Sallis, J.F.; Lo, S.K. Insufficiently Active Australian College Students: Perceived Personal, Social, and Environmental Influences. Preventive Medicine 1999, 28, 20-27, doi:http://dx.doi.org/10.1006/pmed.1998.0375.

7. Sinclair, K.M.; Hamlin, M.J.; Steel, G.D. Physical activity levels of first-year New Zealand university students. Youth Studies Australia 2005, 24, 38-42.

8. Levitsky, D.A.; Halbmaier, C.A.; Mrdjenovic, G. The freshman weight gain: a model for the study of the epidemic of obesity. International Journal of Obesity (London) 2004, 28, 1435-1442.

9. Racette, S.B.; Deusinger, S.S.; Strube, M.J.; Highstein, G.R.; Deusinger, R.H. Weight Changes, Exercise, and Dietary Patterns During Freshman and Sophomore Years of College. Journal of American College Health 2005, 53, 245-251, doi:10.3200/JACH.53.6.245-251.

10. Niemeier, H.M.; Raynor, H.A.; Lloyd-Richardson, E.E.; Rogers, M.L.; Wing, R.R. Fast food consumption and breakfast skipping: predictors of weight gain from adolescence to adulthood in a nationally representative sample. Journal of Adolescent Health 2006, 39, 842-849, doi:10.1016/j.jadohealth.2006.07.001.

11. Adams, T.; Rini, A. Predicting 1-Year Change in Body Mass Index Among College Students. Journal of American College Health 2007, 55, 361-366, doi:10.3200/JACH.55.6.361-366.

12. O'Malley, P.M.; Johnston, L.D. Epidemiology of alcohol and other drug use among American college students. Journal of Studies on Alcohol and Drugs, Supplement 2002, 14, 23-39, doi:doi:10.15288/jsas.2002.s14.23.

13. Singh, A.S.; Mulder, C.; Twisk, J.W.R.; Van Mechelen, W.; Chinapaw, M.J.M. Tracking of childhood overweight into adulthood: a systematic review of the 
literature. Obesity Reviews 2008, 9, 474-488, doi:10.1111/j.1467789X.2008.00475.x.

14. Brevard, P.; Ricketts, C. Residence of College Students Affects Dietary Intake, Physical Activity, and Serum Lipid Levels. Journal of the American Dietetic Association 1996, 96, 35-38, doi:10.1016/s0002-8223(96)00011-9.

15. Maillet, M.A.; Grouzet, F.M.E. Understanding changes in eating behavior during the transition to university from a self-determination theory perspective: a systematic review. Journal of American College Health 2021, 10.1080/07448481.2021.1891922, 1-18, doi:10.1080/07448481.2021.1891922.

16. Australian Bureau of Statistics. Education and Training; Canberra, 2012.

17. Hodge, C.N.; Jackson, L.A.; Sullivan, L.A. The `Freshman 15.'. Psychology of Women Quarterly 1993, 17, 119-126.

18. Butler, S.; Black, D.R.; Blue, C.L.; Gretebeck, R.J. Change in Diet, Physical Activity, and Body Weight in Female College Freshman. American Journal of Health Behavior 2004, 28, 24-32.

19. Baum, C.L. The Effects of College on Weight: Examining the "Freshman 15" Myth and Other Effects of College Over the Life Cycle. Demography 2017, 54, 311-336, doi:10.1007/s13524-016-0530-6.

20. Vadeboncoeur, C.; Townsend, N.; Foster, C. A meta-analysis of weight gain in first year university students: is freshman 15 a myth? BMC Obesity 2015, 2, 22, doi:10.1186/s40608-015-0051-7.

21. Lloyd-Richardson, E.E.; Bailey, S.; Fava, J.L.; Wing, R. A prospective study of weight gain during the college freshman and sophomore years. Preventive Medicine 2009, 48, 256-261, doi:http://dx.doi.org/10.1016/j.ypmed.2008.12.009.

22. Miller, K.G.; Hartman, J.M. Influence of physical activity on weight status during the first year of college. Journal of American College Health 2020, 68, 258-262, doi:10.1080/07448481.2018.1539398.

23. International Society for the Advancement of Kinanthropometry. International Standards for Anthropometric Assessment. International Society for the Advancement of Kinanthropometry: New Zealand, 2011; pp 52-81.

24. Gupta, N.; Balasekaran, G.; Govindaswamy, V.V.; Hwa, C.Y.; Shun, L.M. Comparison of body composition with bioelectric impedance (BIA) and dual energy X-ray absorptiometry (DEXA) among Singapore Chinese. Journal of Science and Medicine in Sport 2011, 14, 33-35.

25. Batterham, M.; Tapsell, L.; Jenkins, A. A comparison of bioelectrical impedance and near infra-red interactance with dual energy x-ray absorptiometry for the determination of body fat. Nutrition Dietetics 2002, 59, 120-126. 
26. Xyris Software Australia Pty Ltd FoodWorks 8 Professional, 8; Xyris Software Australia Pty Ltd: Australia, 2015.

27. National Health and Medical Research Council. Eat for Health: Australian Dietary Guidelines; National Health and Medical Research Council: Canberra, 2013; pp 1-226.

28. Craig, C.L.; Marshall, A.L.; Sjostrom, M.; Bauman, A.E.; Booth, M.L.; Ainsworth, B.E.; Pratt, M.; Ekelund, U.; Yngve, A.; Sallis, J.F., et al. International physical activity questionnaire: 12 -country reliability and validity. Medicine and science in sports and exercise 2003, 35, 1381-1395, doi:10.1249/01.MSS.0000078924.61453.FB.

29. Ainsworth, B.E.; Haskell, W.L.; Leon, A.S.; Jacobs, D.R.J.; Montoye, H.J.; Sallis, J.F.; Paffenbarger, R.S.J. Compendium of Physical Activities: classification of energy costs of human physical activities. Medicine \& Science in Sports \& Exercise 1993, 25, 71-80.

30. Crouter, S.; Antczak, A.; Hudak, J.; DellaValle, D.; Haas, J. Accuracy and reliability of the ParvoMedics TrueOne 2400 and MedGraphics VO2000 metabolic systems. European Journal of Applied Physiology. 2006, 98, 139-151, doi:10.1007/s00421-006-0255-0.

31. Weir, J.B.d.V. New methods for calculating metabolic rate with special reference to protein metabolism. The Journal of Physiology 1949, 109, 1-9, doi:10.1113/jphysiol.1949.sp004363.

32. Stunkard, A.J.; Messick, S. The three-factor eating questionnaire to measure dietary restraint, disinhibition and hunger. Journal of Psychosomatic Research 1985, 29, 71-83, doi:http://dx.doi.org/10.1016/0022-3999(85)90010-8.

33. IBM Statistical Product and Service Solution (SPSS) Statistics, , 21; IBM: Chicago, USA, 2012.

34. Hajhosseini, L.; Holmes, T.; Mohamadi, P.; Goudarzi, V.; McProud, L.; Hollenbeck, C.B. Changes in Body Weight, Body Composition and Resting Metabolic Rate (RMR) in First-Year University Freshmen Students. Journal of the American College of Nutrition 2006, 25, 123-127, doi:10.1080/07315724.2006.10719522.

35. Finlayson, G.; Cecil, J.; Higgs, S.; Hill, A.; Hetherington, M. Susceptibility to weight gain. Eating behaviour traits and physical activity as predictors of weight gain during the first year of university. Appetite 2012, 58, 1091-1098, doi:http://dx.doi.org/10.1016/j.appet.2012.03.003.

36. Deliens, T.; Clarys, P.; Van Hecke, L.; De Bourdeaudhuij, I.; Deforche, B. Changes in weight and body composition during the first semester at university. A prospective explanatory study. Appetite 2013, 65, 111-116, doi:http://dx.doi.org/10.1016/j.appet.2013.01.024. 
37. Mifsud, G.; Duval, K.; Doucet, E. Low body fat and high cardiorespiratory fitness at the onset of the freshmen year may not protect against weight gain. British Journal of Nutrition 2009, 101, 1406-1412, doi:10.1017/S0007114508067639.

38. Anderson, D.A.; Shapiro, J.R.; Lundgren, J.D. The freshman year of college as a critical period for weight gain: An initial evaluation. Eating behaviors 2003, 4, 363-367, doi:http://dx.doi.org/10.1016/S1471-0153(03)00030-8.

39. Levitsky, D.A.; Garay, J.; Nausbaum, M.; Neighbors, L.; Dellavalle, D.M. Monitoring weight daily blocks the freshman weight gain: a model for combating the epidemic of obesity. International Journal of Obesity (London) 2006, 30, 1003-1010, doi:10.1038/sj.ijo.0803221.

40. Kęska, A.; Lutosławska, G.; Mazurek, K.; Czajkowska, A.; Tkaczyk, J.; Iwańska, D. Changes in Anthropometry and Selected Metabolic Parameters in Young Men During Their First Year of Study at a University of Physical Education. American Journal of Men's Health 2017, 12, 463-471, doi:10.1177/1557988317743151.

41. Jung, M.E.; Bray, S.R.; Martin Ginis, K.A. Behavior Change and the Freshman 15: Tracking Physical Activity and Dietary Patterns in 1st-Year University Women. Journal of American College Health 2008, 56, 523-530.

42. Olansky, S.; Beaudry, K.M.; Woods, S.; Barbour-Tuck, E.; Gammage, K.L.; Klentrou, P.; Josse, A.R. Changes in Body Composition, Energy Expenditure, and Energy Intake during Four Years of University-A Follow-Up Study. International Journal of Environmental Research and Public Health 2021, 18, 3990.

43. Costa, C.; Moreira, P.; Teixeira, V. Holiday weight gain in university students. Alimentação Humana 2007, 13, 17-19.

44. Tayyem, R.F.; Bayadoo, F.; Kassab, M.; Khriesat, W.M.; Al-Haifi, A.R.; Banks, A.D.; Bawadi, H.A. Association of college entrance exam with changes in some lifestyle factors among adolescents. Nutrition \& Food Science 2013, 43, 573581, doi:doi:10.1108/NFS-09-2012-0100.

45. Sprake, E.; Lavin, J.; Grabowski, P.; Russell, J.; Featherstone, M.; Barker, M. Eating habits associated with body weight gain in female university students: A UK-based study of Slimming World members. British Food Journal 2017, 119, 2571-2582, doi:10.1108/BFJ-10-2016-0495.

46. Economos, C.D.; Hilderbrandt, M.L.; Hyatt, R.R. College Freshman Stress and Weight Change: Differences by Gender. American Journal of Health Behaviour 2008, 32, 16-25.

47. Du, C.; Luo, N.; Wu, L.; Gadd, S.; Zhang, X.; Tucker, R.M. Health behavior changes associated with weight gain among first-year international students 
studying at an American university. Journal of American College Health 2021, 10.1080/07448481.2021.1891082, 1-10, doi:10.1080/07448481.2021.1891082.

48. Pullman, A.W.; Masters, R.C.; Zalot, L.C.; Carde, L.E.; Saraiva, M.M.; Dam, Y.Y.; Randall Simpson, J.A.; Duncan, A.M. Effect of the transition from high school to university on anthropometric and lifestyle variables in malesPresented in part at the Canadian Society for Nutritional Sciences, Canadian Nutrition Congress, held in Winnipeg, Manitoba, from 18-21 June 2007. Applied Physiology, Nutrition, and Metabolism 2009, 34, 162-171, doi:10.1139/H09007.

49. Jebb, S.A.; Moore, M.S. Contribution of a sedentary lifestyle and inactivity to the etiology of overweight and obesity: current evidence and research issues. Medicine and science in sports and exercise 1999, 31, S534-541, doi:10.1097/00005768-199911001-00008.

50. De Cocker, K.A.; van Uffelen, J.G.Z.; Brown, W.J. Associations between sitting time and weight in young adult Australian women. Preventive Medicine 2010, 51, 361-367, doi:http://dx.doi.org/10.1016/j.ypmed.2010.07.009.

51. van der Ploeg, H.P.; Chey, T.; Korda, R.J.; Banks, E.; Bauman, A. Sitting time and all-cause mortality risk in 222,497 Australian adults. Archives of Internal Medicine 2012, 172, 494-500, doi:10.1001/archinternmed.2011.2174.

52. Wilmot, E.G.; Edwardson, C.L.; Achana, F.A.; Davies, M.J.; Gorely, T.; Gray, L.J.; Khunti, K.; Yates, T.; Biddle, S.J. Sedentary time in adults and the association with diabetes, cardiovascular disease and death: systematic review and meta-analysis. Diabetologia 2012, 55, 2895-2905.

53. Cluskey, M.; Grobe, D. College Weight Gain and Behavior Transitions: Male and Female Differences. Journal of the American Dietetic Association 2009, 109, 325-329, doi:http://dx.doi.org/10.1016/j.jada.2008.10.045.

54. Papadaki, A.; Hondros, G.; A. Scott, J.; Kapsokefalou, M. Eating habits of University students living at, or away from home in Greece. Appetite 2007, 49, 169-176, doi:http://dx.doi.org/10.1016/j.appet.2007.01.008.

55. Vella-Zarb, R.A.; Elgar, F.J. Predicting the 'freshman 15': Environmental and psychological predictors of weight gain in first-year university students. Health Education Journal 2010, 69, 321-332, doi:10.1177/0017896910369416.

56. Nikolaou, C.K.; Hankey, C.R.; Lean, M.E. Weight changes in young adults: a mixed-methods study. International Journal of Obesity (London) 2015, 39, 508513, doi:10.1038/ijo.2014.160.

57. Yakusheva, O.; Kapinos, K.; Weiss, M. Peer effects and the Freshman 15: Evidence from a natural experiment. Economics \& Human Biology 2011, 9, 119-132, doi:http://dx.doi.org/10.1016/j.ehb.2010.12.002. 
58. Rocks, T.; Pelly, F.; Slater, G.; Martin, L.A. Eating attitudes and behaviours of students enrolled in undergraduate nutrition and dietetics degrees. Nutrition \& Dietetics 2016.

59. Hootman, K.C.; Guertin, K.A.; Cassano, P.A. Stress and psychological constructs related to eating behavior are associated with anthropometry and body composition in young adults. Appetite 2018, 125, 287-294, doi:https://doi.org/10.1016/j.appet.2018.01.003.

60. World Health Organization. Obesity and overweight. Availabe online: http://www.who.int/mediacentre/factsheets/fs311/en/ (accessed on 12/06/15).

61. Elmadfa, I.; Kornsteiner, M. Fats and Fatty Acid Requirements for Adults. Annals of Nutrition and Metabolism 2009, 55, 56-75.

62. National Health and Medical Research Council. Sodium: Nutrient Reference Values for Australia and New Zealand Including Recommended Dietary Intakes; National Health and Medical Research Council: Canberra, 2017; pp 209-216.

63. Strazzullo, P.; D’Elia, L.; Kandala, N.-B.; Cappuccio, F.P. Salt intake, stroke, and cardiovascular disease: meta-analysis of prospective studies. British Medical Journal 2009, 339, 1-9, doi:10.1136/bmj.b4567.

64. World Health Organization. Guideline: Sugars intake for adults and children; WHO Press: Geneva, 2015; pp 16-17.

65. World Health Organization. Diet, nutrition and the prevention of chronic diseases: report of a joint WHO/FAO expert consultation; World Health Organization: Geneva, 2003; pp 1-149.

66. Hilger, J.; Loerbroks, A.; Diehl, K. Eating behaviour of university students in Germany: Dietary intake, barriers to healthy eating and changes in eating behaviour since the time of matriculation. Appetite 2017, 109, 100-107, doi:https://doi.org/10.1016/j.appet.2016.11.016.

67. Pinto Guedes, D.; dos Santos Silva, A.L.; Guedes, D.P.; Silva, A.L.D.S. Ejercicio físico y consumo de frutas/verduras, y sus asociaciones con el estado del peso corporal en estudiantes universitarios. Nutricion Hospitalaria 2021, 38, 545-554, doi:10.20960/nh.03258.

68. Kyle, U.G.; Bosaeus, I.; De Lorenzo, A.D.; Deurenberg, P.; Elia, M.; Manuel Gómez, J.; Lilienthal Heitmann, B.; Kent-Smith, L.; Melchior, J.-C.; Pirlich, M., et al. Bioelectrical impedance analysis - part II: utilization in clinical practice. Clinical Nutrition 2004, 23, 1430-1453, doi:http://dx.doi.org/10.1016/j.clnu.2004.09.012. 